\title{
The development and water-air two-phase test results of an electrical submersible pump visualization prototype
}

\author{
F. E. Trevisan \& M. G. Prado \\ Department of Petroleum Engineering, University of Tulsa, USA
}

\begin{abstract}
Artificial lift systems are extensively used in the petroleum industry in order to enhance or even initiate the production of oil wells that present insufficient energy to produce naturally. The Electrical Submersible Pump (ESP) is one of the many existing artificial lift techniques and consists of assembling several diffuser type centrifugal pumps in series.

It is common in petroleum field applications to have an inflow of natural gas into the pump intake, which compromises pump performance and reliability. Trying to better understand the interaction between the gas and liquid inside centrifugal pumps is still a challenge and the current investigation tries to help understand this subject.

This research describes the development of a visualization prototype built using original ESP components, applying minimal mechanical and geometrical modifications, with the intent of acquiring visual evidence of the flow patterns that occur inside the pump. Because of these minimal changes to the original pump geometry, the prototype was proven to operate according to the original pump catalogue performance, implying that its hydraulic behaviour was maintained.

Water-air two phase experiments were conducted at different rotational speeds $(15,20,30 \mathrm{~Hz})$ and non-slip void fractions (up to $5 \%$ ) while the liquid rate was kept constant at $60 \%$ of the maximum rate at the defined shaft speed. High speed video footage was gathered, as well as differential pressure measurements.

The authors identified four water-air flow patterns inside the impeller channels: Dispersed Bubbles, Agglomerated Bubbles, Gas Pocket and Segregated Gas. By comparing the images with the differential pressure data, it
\end{abstract}


was confirmed that pump two-phase homogeneous flow head prediction is quite accurate under the Dispersed Bubble pattern. It was concluded that the Agglomerated Bubble pattern is responsible for the initial head degradation and that the surging event coincides with the Gas Pocket structure, indicating that this is an interface instability problem.

Keywords: centrifugal pump, flow visualization, two-phase flow, surging, flow patterns.

\section{Introduction}

The Electrical Submersible Pump (ESP) is one of the many existing artificial lift techniques used in the petroleum industry. It consists of a series of small diameter diffuser type casing centrifugal pumps. ESPs are mainly known for their capacity of handling high volumes and are widely used. Their applications vary from producing high productivity oil wells onshore and offshore to dewatering coal bed methane and gas wells.

The use of ESPs, or any other centrifugal pump for that matter, on liquid single phase applications is well understood and the knowledge necessary for these situations is consolidated. However, as is common in the petroleum industry, the presence of a compressible phase, such as natural gas, will also modify the hydraulic performance of these types of pump.

Several studies have been conducted to visualize and simulate pump performance under the presence of free gas; the majority of these studies focus on centrifugal pumps used by the nuclear industry, motivated by the flux of water and water vapour in their systems. Murakami and Minemura [1] used a semi-open type pump with transparent casing. The observed flow patterns were recorded photographically, and bubble diameter measurements could be made inside the impeller. Patel et al. [2] also observed two-phase flow in centrifugal pumps by testing a $1 / 20$ scale prototype of a primary coolant pump used in nuclear reactors, to measure bubble sizes. Sekoguchi et al. [3] used a pump equipped with a closed radial-flow impeller to visualize the flow patterns and also installed eight needle tip void probes to measure bubble frequency and later examined the slip ratio between the fluids.

ESPs, however, are different from these pumps, they are smaller in diameter, they are diffuser-type rather than volute-type design and consist of several stages, whereas single-staged pumps are used in the nuclear industry. ESP twophase investigations started with Lea and Bearden [4] by performing tests on three different impeller designs, two radial and one mixed flow type. The authors also used two different fluid combinations, first water and air and later diesel oil and carbon dioxide. Sachdeva [5] proposed a one-dimensional model for single and two-phase performance of an ESP. In his work, derivation of the governing equations was done and later solved numerically. Pessoa [6] published an extensive experimental investigation with two-phase flow performance of a mixed flow type impeller ESP. Duran [7] performed two-phase experiments on an Electrical Submersible Pump with water. The objective of the experiments 
was to measure the performance of a specific stage, observing its pressure increment at several stage intake pressures and water and air flow rates.

More recently, flow modelling complemented by visualization in these types of centrifugal pumps have been investigated by Estevan [8] who used an ESP impeller and a modified Plexiglas diffuser to observe air-water flow inside the pump. The author noticed that accumulation of gas in the pressure side of the blade and two distinct flow patterns occurring at different sections of the impeller. Estevan used the visual observations to develop a one-dimensional model. Barrios [9] also built a visualization prototype based on an ESP impeller and modified diffuser design. The author used a high speed camera to observe the flow patterns and take bubble size measurements in order to complete the proposed one-dimensional model. Later, Gamboa [10] built a visualization prototype to obtain images used to explain the hydraulic behaviour and illustrate the flow pattern map developed through pressure generation data, collected in a large scale experiment. Both pumps used had the same geometrical properties; the visualization prototype was built by minimally altering the large scale pump stages. However, because of this minimal alteration, the author could only observe the impeller discharge and diffuser intake.

These previous work indicated that there was a trade-off between visualization and geometrical integrity. Therefore the objective of this work was to develop a visualization prototype that allowed two-phase flow visualization inside the impeller with minimum alteration on the pump geometrical properties, thus unchanging its hydraulic performance.

By building such prototype water single phase tests were conducted to validate this premise. Water-air two-phase flow tests were later conducted where pressure generation data and high speed video footage of the flow patterns inside the impeller were collected.

\section{Experimental description}

\subsection{Prototype development}

The visualization prototype had the primary intent of imitating the hydraulic behaviour of an actual ESP. This could only be obtained by introducing minimal geometrical alterations to actual ESP stages while installing visualization windows. The original pump stage from which the prototype was developed was the Baker Huges Centrilift FC1600 model. It was chosen for its large radius, which provided reasonable viewing area, and for its low capacity, which respected flow loop limitations. Figure 1 presents a sketch of proposed alterations.

It can be observed that only the entrance (lower section) of the top diffuser was modified. Figure 2 presents the development of this component; on the left is the original diffuser, in the middle this piece is shown with the necessary alterations and on the right it is shown with the Plexiglas installed. The lower external walls were machined off, exposing the diffuser's internal blades and channels entrance. The Plexiglas piece installed recreated the original external wall geometry and accommodated the upper mechanical seal. 


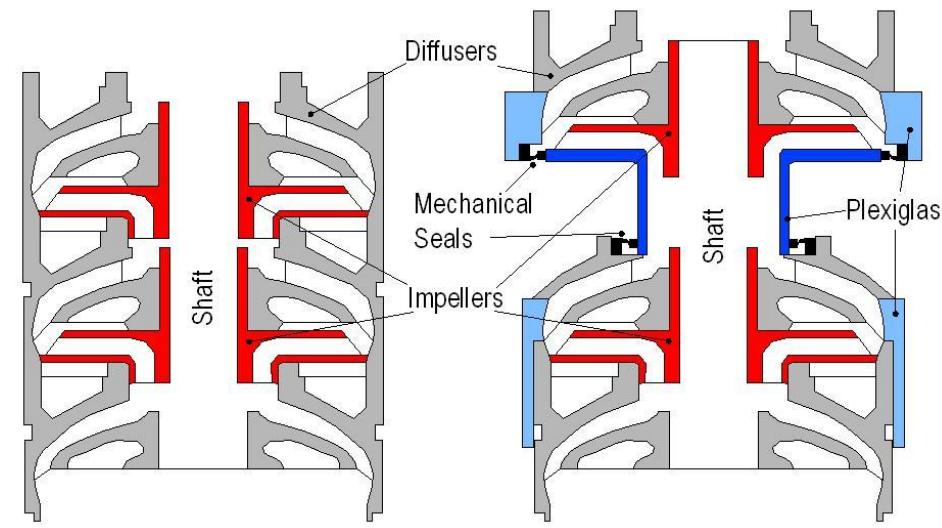

Figure 1: $\quad$ Original ESP (left) modified ESP (right).
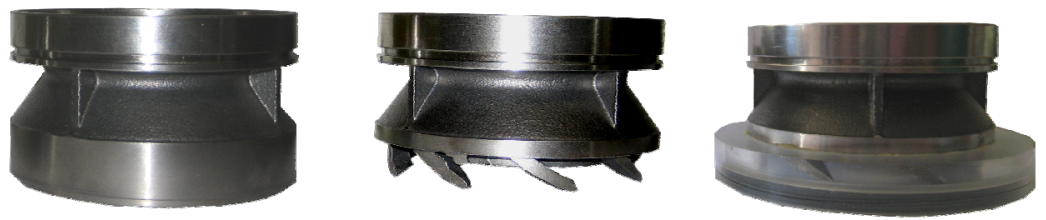

Figure 2: $\quad$ Top diffuser development process.
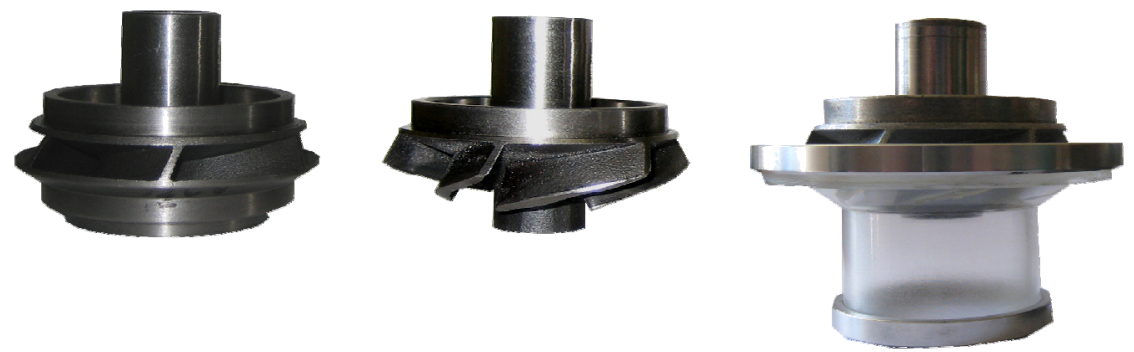

Figure 3: Impeller development process.

Figure 3 presents the impeller development process. On the left is the original piece, in the middle is the impeller with its bottom shroud removed and on the right the piece is shown with the Plexiglas installed, with the mechanical seals' stainless steel rings in place. The original impeller entrance diameter and the original shroud angles were preserved when building the Plexiglas attachment.

The bottom diffuser had the most modifications of all components. In order to accommodate the lower mechanical seal and improve the visualization window, its top section was removed and internal diameter enlarged, however the internal channels fluid discharge remained intact. The external wall was machined off, exposing the lower sections of the diffuser blades and channels; however, the 
Plexiglas window installed on the lower part of this component had to accommodate the impeller and diffuser from the stage bellow. Figure 4 presents the original diffuser and in its final state.

The entrance diffuser assembly process is presented in Figure 5, where an unaltered stage was assembled prior to the visualization stage.

The mechanical seals used were custom built for this application. They consisted of a stainless steel ring, installed on the top and bottom of the impeller Plexiglas shroud, and a lip seal design made out of PolyShell ${ }^{\mathbb{B}}-03$ (graphite filled PTFE) placed at the top diffuser entrance and bottom diffuser discharge.

In order to hold all the components sealed in place and connected to the stand that held the motor, a PVC casing was machined for both upper and lower diffusers. Figure 6 presents a sketch of these components as well as the pressure taps location used for head generation data collection. This information was collected only for the visualization stage (between taps PT1 and PT2).

\subsection{Experimental loop and procedure}

In order to make the necessary measurements, an ESP visualization prototype was installed in a closed circuit loop. Figure 7 provides a graphical representation of the experimental facility built.
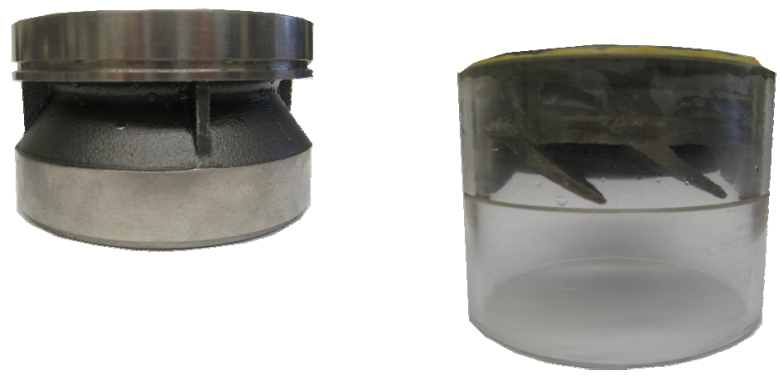

Figure 4: Bottom diffuser development process.
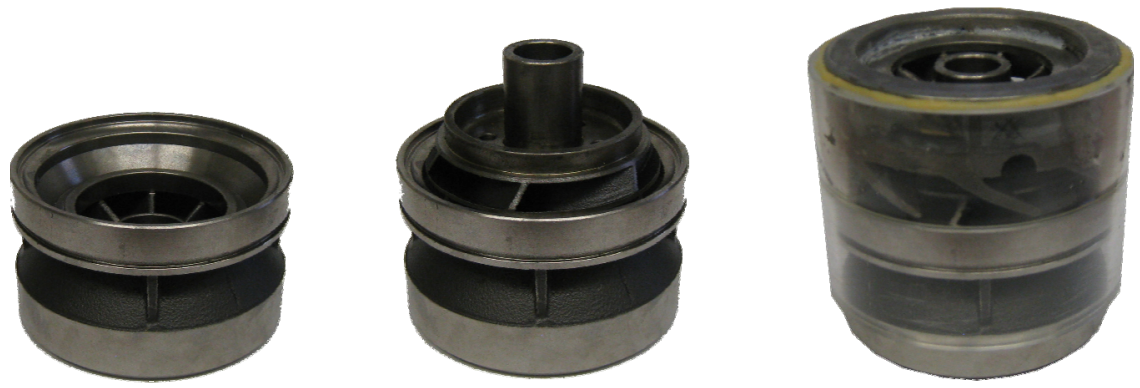

Figure 5: Entrance stage assembly process. 

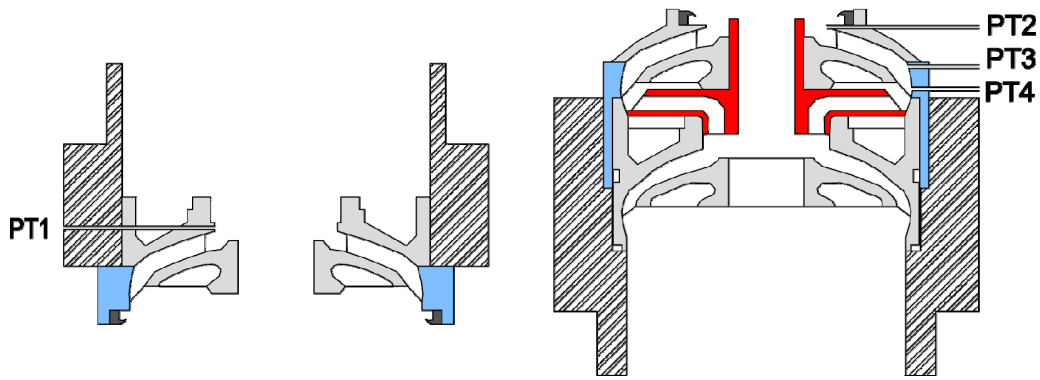

Figure 6: Prototype PVC casing sketch, upper section (left) and lower section (right).

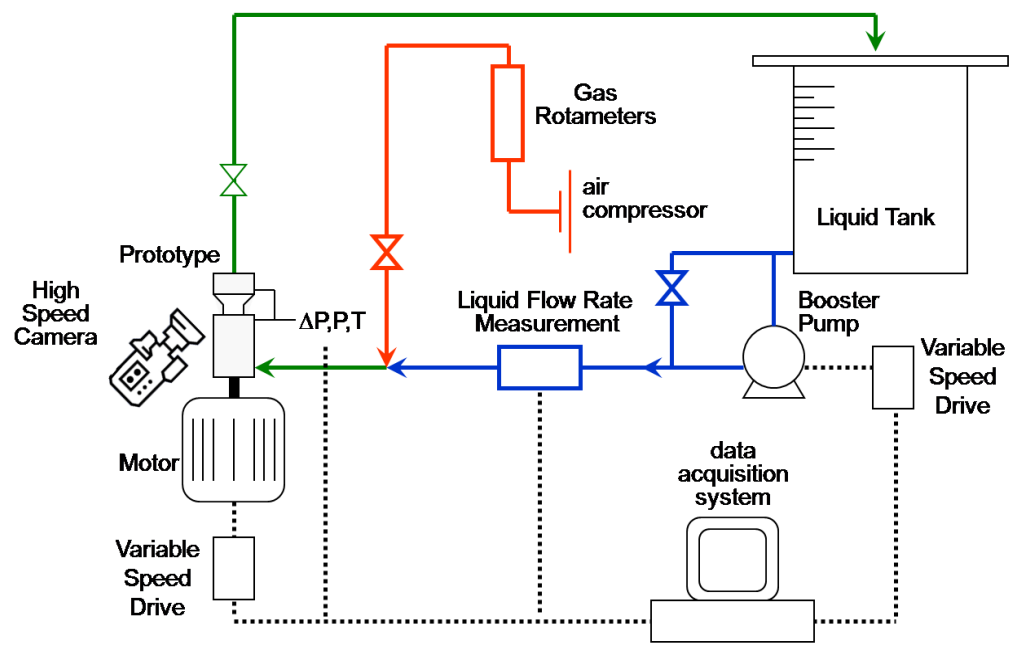

Figure 7: Visualization loop configuration.

The two-phase testing procedure was based on previous experience and followed the so called "Surging Test" method, in which the pump rotational speed, visualization stage inlet pressure and liquid flow rate are kept constant while the gas flow rate is increased and the pressure increment generated by the visualization stage is measured. The liquid flow rate used for each test was determined as $60 \%$ of the maximum liquid rate at the specified shaft speed. This value is commonly used in the industry as a high efficiency pump operational point.

The non-slip void fraction, given by the ratio between the gas flow rate and the total mixture flow rate, varied from 0 to $5 \%$. Three different shaft speeds were used during the two-phase tests: 15, 20 and $30 \mathrm{~Hz}$. 


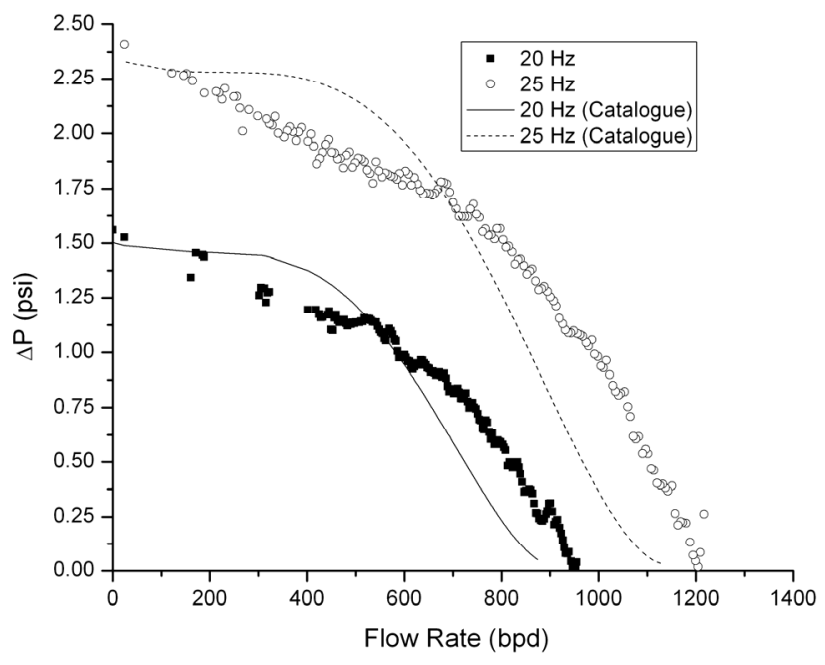

Figure 8: $\quad$ Prototype versus catalogue performance.

\section{Results and analysis}

\subsection{Single-phase tests}

The first experiments conducted were single-phase tests at two different rotational speeds: 20 and $25 \mathrm{~Hz}$. The purpose of these experiments was to characterize the pump, comparing it to its original design catalogue behaviour.

From the comparison presented in Figure 8, it can be seen that the prototype presents a similar behaviour as the original pump, especially at the shut-in pressure and at values around the $60 \%$ of the maximum flow rate. However, some minor differences can be observed which may be explained by the different recirculation patterns inside original ESP and prototype, especially because the first presents a floating impeller and the second a fixed impeller.

Shut-in and open flow single phase tests were also conducted with the intent to prove that the prototype obeyed the Affinity Laws, thus proving that it operated as a centrifugal device. Figure 9 presents the experimental points as well as the quadratic fit line and linear fit line found for the shut-in pressures and maximum flow rates, respectively.

\subsection{Two-phase tests}

Based on the high speed video footage taken during the water and air two-phase flow tests, four flow patterns were identified and their descriptions are as follows.

Bubbly Flow: At low gas flow rates, independent of the liquid rate or rotational speed, this pattern was observed. It consists of small air bubbles rising 


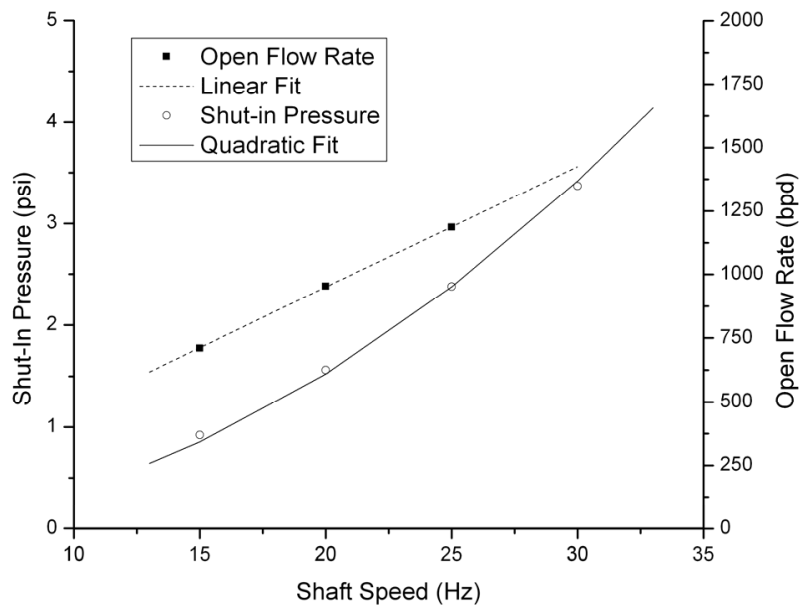

Figure 9: Water shut-in and maximum flow rates versus rotational speed.

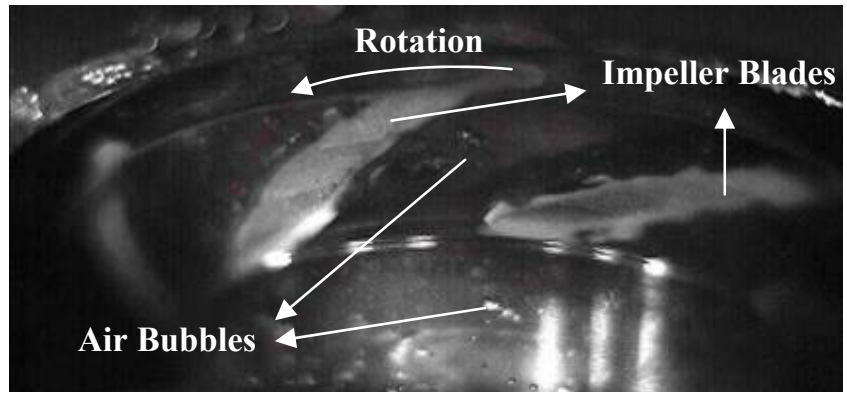

Figure 10: Bubbly flow pattern.

through the impeller entrance and being redirected when entering the impeller channels. Figure 10 presents a still image obtained from the high speed video.

The gas rate influenced mainly the quantity of bubbles observed, while their size and shape varied from spherical for smaller entities to prolate ellipsoidal for larger units. Eventually even larger bubbles were seen with an irregular shape rising through the impeller entrance; however, they would break up into smaller units upon reaching the impeller channel.

Agglomerated Bubbles: This flow pattern was obtained by increasing the gas flow rate once at the bubbly flow pattern. It is characterized by the presence of agglomerated bubbles at the impeller blade leading end and pressure side. Figure 11 presents the impeller channels under this flow pattern.

Gas Pocket: By further increasing the gas flow rate, it was noticed that the gas pocket formed by bubble coalescence from the previous flow structure. This larger stationary entity occupied a significant section of the impeller channel, restricting the liquid's flow path. The stationary bubble gas-liquid interface was 


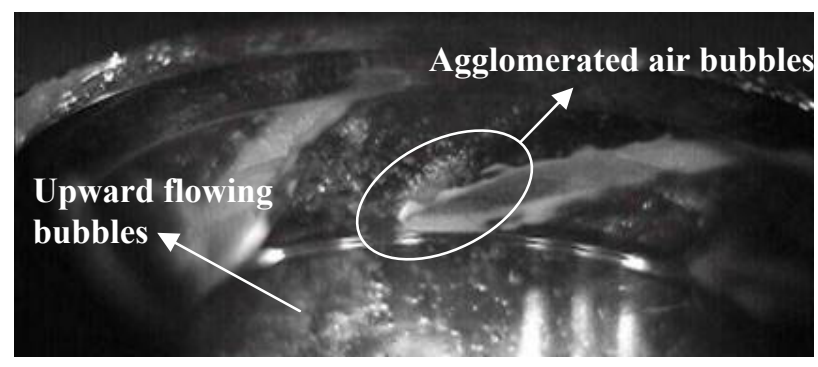

Figure 11: Agglomerated bubbles.

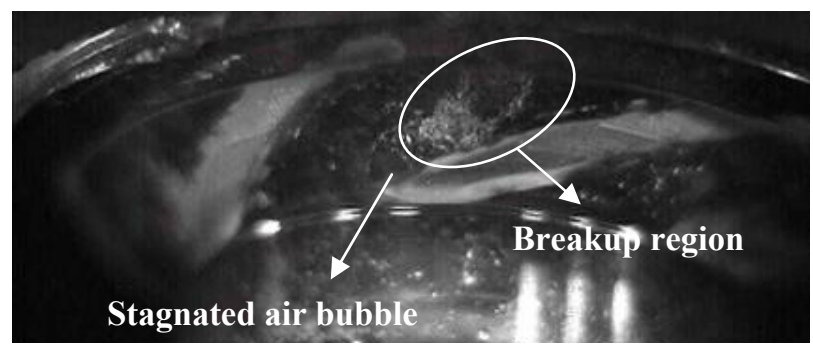

Figure 12: Gas pocket pattern.

observed to be unsteady, indicating instability. Gas bubbles rising through the impeller entrance were dragged with the water through its reduced flow area or coalesced with the stationary gas pocket which led to break up at its trailing end. Figure 12 presents a photograph of this observed pattern.

It was also observed that some larger bubbles, with irregular shape, rose through the impeller entrance. However, in this case, instead of breaking up as in previous cases, they either coalesced with the gas pocket or passed through the impeller channel intact. This indicates that the stationary gas bubble reduces the kinetic energy transferred to the liquid, which causes the larger bubbles not to break up.

Segregated Gas: By further increasing the gas flow rate, it was possible to observe that the gas pocket had been extended to the impeller outer radius. As before, the gas-liquid interface remained unstable, indicating bubble break up at the stationary element's trailing end. Figure 13 presents the described flow structure.

By following the experimental procedure described previously, differential pressure data was collected for the surging tests at rotational speeds of 15, 20 and $30 \mathrm{~Hz}$. Figure 14 presents the information collected by plotting on the horizontal axis the no-slip void fraction and on the vertical axis the normalized differential pressure.

This figure shows the effect of rotational speed on the pump two-phase performance. At low void fraction values, all curves present relatively small deviations from its homogeneous flow prediction, since at these gas rates the air 


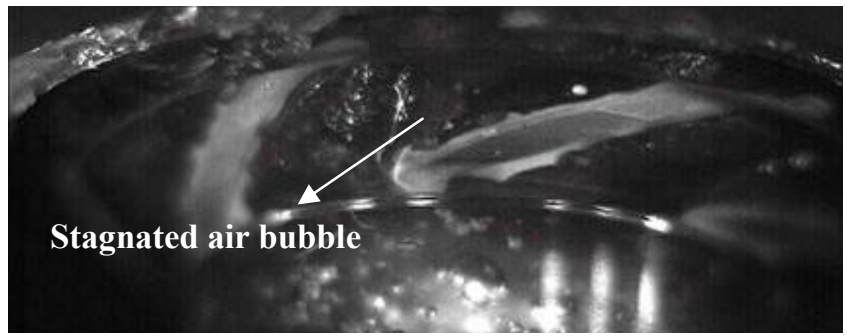

Figure 13: $\quad$ Segregated gas pattern.

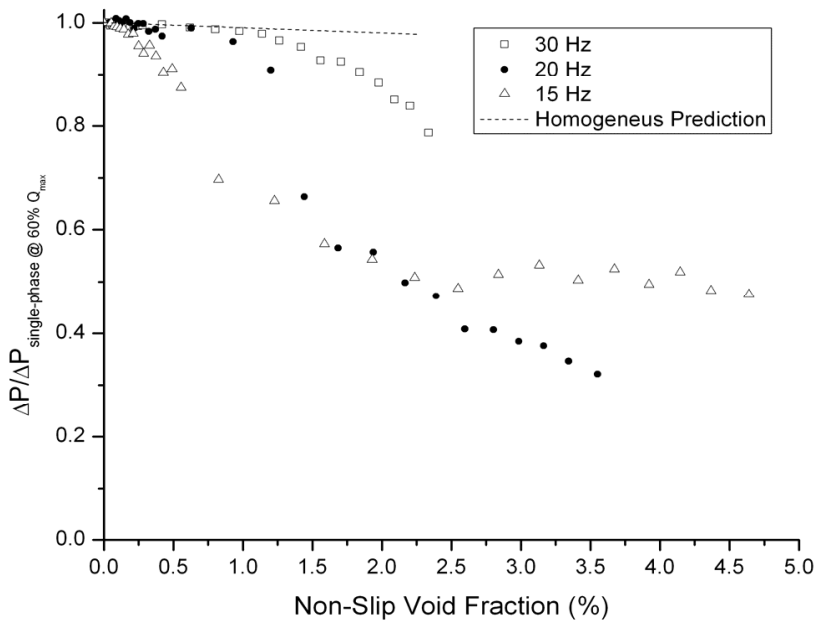

Figure 14: Water-air surging test results.

bubbles are predominantly small and flow with the liquid. By increasing air injection, a non-linear, but continuous, deviation from the homogeneous prediction is observed. Further increase of the no-slip gas fraction caused the surging phenomenon to occur, indicated by the curves sudden inclination change and in this case seen as a discontinuity. It can be observed that at 15 and $20 \mathrm{~Hz}$ the surging occurs at void fractions of 0.55 and $1.3 \%$, respectively, while on the $30 \mathrm{~Hz}$ curve surging was not reached.

By combining the same plot style as Figure 14 with the flow pattern identification used previously, Figure 15 was obtained. This graph indicates adequate association of the Bubbly Flow pattern with the homogeneous model prediction. It can also be observed that the Agglomerated Bubbles pattern, where slip between the phases was observed, is strongly associated with head degradation thus deviation from the homogeneous predictions.

It can also be seen that the two occurrences of the Gas Pocket pattern correspond to void fractions of 0.55 and 1.44 for rotational speeds of 15 and 


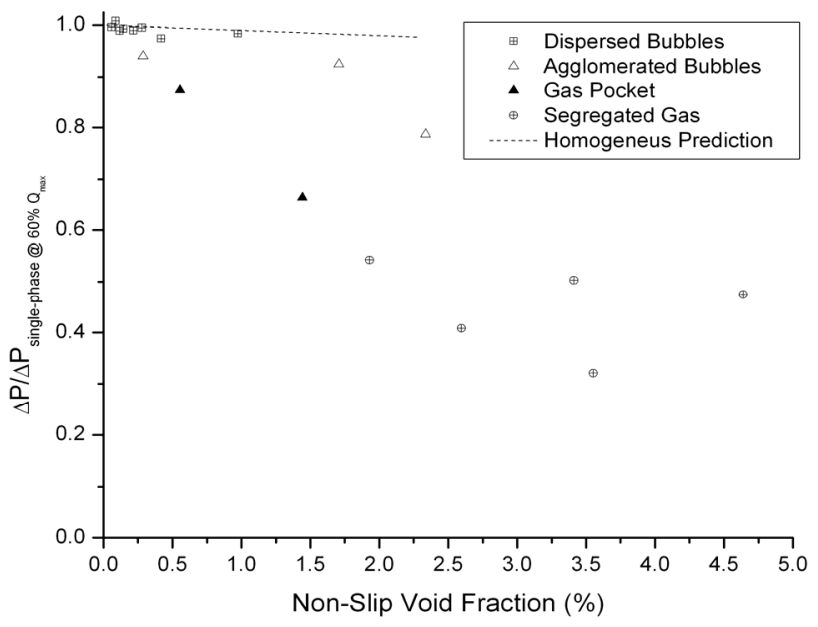

Figure 15: Flow pattern occurrence on data.

$20 \mathrm{~Hz}$, respectively, previously identified as surging points. However, for $15 \mathrm{~Hz}$ it corresponds to a pre-surging state, while for $25 \mathrm{~Hz}$ for a post-surging condition. This indicates that this pattern holding the pre-surging differential pressure is an unstable equilibrium point. Thus, the surging phenomenon is a shift on the equilibrium position to a lower differential pressure value, allowing the same flow pattern to occur at two different differential pressure conditions.

The Segregated Gas flow structure is responsible for the most head degradation measured. In this case, the stationary gas bubble alters the impeller geometry seen by the liquid, drastically depredating performance.

\section{Conclusions}

From this research the following conclusions could be made: a visualization prototype was successfully built with minor geometrical alterations to the original ESP design, imposing a very similar hydraulic performance; the liquid turbulence maintains small air bubble diameters, which are dragged with the water through the impeller; slip between the phases occurs in the Agglomerated Bubbles pattern, observed in the video footage and in head degradation data; surging phenomenon occurs in the Gas Pocket pattern. The images and data indicate that it is a transient phenomenon event and probably do to interfacial instability.

\section{Acknowledgements}

The present work was supported by CNPq, a Brazilian Government entity dedicated to scientific and technological development. The authors also would like to thank Baker Hughes Centrilift for their material and technical support. 


\section{References}

[1] Murakami and Minemura. Effects of Entrained Air on the performance of a centrifugal pump. Bulletin of the JSME., Vol. 17, No 110, August $1974\left(1^{\text {st }}\right.$ report).

[2] Patel, Bharatan and Runstadler, Peter Jr. Investigations into the Two-Phase Behavior of Centrifugal Pumps. Polyphase Flow in Turbomach, Symp presented at Winter Annual Meeting of the ASME. Dec 10-15 1978.

[3] Sekoguchi K., Takada S and Kanemori Y. Study of Air-Water Two-Phase Centrifugal Pump by Means of Electric Resistivity Probe Technique for Void Fraction Measurement ( $1^{\text {st }}$ Report). Bulletin of JSME. Vol. 27, No 227, May 1984.

[4] Lea J. F. and J. L. Bearden. "Effect of Gaseous Fluids on Submersible Pumps" paper presented at SPE 55th Annual Fall Technical Conference and Exhibition, SPE 9218, (September 1980).pp: 2922-2930.

[5] Sachdeva R. Performance of electrical Submersible pump in Gassy Wells. SPE Paper. 22767. 55.

[6] Pessoa, R. "Experimental Investigation of Two-Phase Flow Performance of Electrical Submersible Pump Stages", MS Thesis, The University of Tulsa (2001).

[7] Duran, Javier. "Pressure Effects on ESP Stages' Air-Water Performance", M.Sc. Thesis, The University of Tulsa (2003)

[8] ESTEVAM, Valdir. A Phenomenological Analysis about centrifugal pump in two-phase flow operation. Campinas: Faculdade de Engenharia Mecânica, Universidade Estadual de Campinas, 2002. 265 p. Thesis (PhD)

[9] Barrios, Lisset. "Visualization and Modelling of Multiphase Performance inside an ESP". PhD Dissertation, The University of Tulsa (2007)

[10] Gamboa, Jose. "Prediction of the Transition in Two-Phase Performance of an Electrical Submersible Pump". PhD Dissertation, The University of (2008) 